\title{
Linear perturbations of spatially locally homogeneous spacetimes
}

\author{
Masayuki Tanimoto
}

\begin{abstract}
Methods and properties regarding the linear perturbations are discussed for some spatially closed (vacuum) solutions of Einstein's equation. The main focus is on two kinds of spatially locally homogeneous solution; one is the Bianchi III (Thurston's $H^{2} \times \mathbf{R}$ ) type, while the other is the Bianchi II (Thurston's Nil) type. With a brief summary of previous results on the Bianchi III perturbations, asymptotic solutions for the gauge-invariant variables for the Bianchi III are shown, with which (in)stability of the background solution is also examined. The issue of linear stability for a Bianchi II solution is still an open problem. To approach it, appropriate eigenfunctions are presented for an explicitly compactified Bianchi II manifold and based on that, some field equations on the Bianchi II background spacetime are studied. Differences between perturbation analyses for Bianchi class B (to which Bianchi III belongs) and class A (to which Bianchi II belongs) are stressed for an intention to be helpful for applications to other models.
\end{abstract}

\section{Introduction}

A classification of three dimensional homogeneous (Riemannian) manifolds is known in relativity as the Bianchi classification, which is a classification of the three dimensional simply transitive Lie groups by isomorphism. A four dimensional spacetime $\left(X \times \mathbf{R}, \mathrm{g}_{a b}\right)$ is said to be spatially homogeneous if a Bianchi group $G$ acts spacelike and makes the metric $g_{a b}$ invariant. Cosmological models based on such a spacetime structure, especially with isotropy, have long been a basis for cosmology, and (in case of being anisotropic) have recently been subjects for more mathematical study in relativity (e.g. WE ). Departure from a homogeneity however belongs to a very recent development in the field (except for linear perturbations of rather special homogeneous solutions such as those with isotropy or ones that are not isotropic but can become so in a limit). In particular, whether the solution is stable or not is unknown for almost all such solutions.

2000 Mathematics Subject Classification. Primary 83C25; Secondary 53C50, 58J37.

Key words and phrases. gauge-invariant perturbations, cosmological models, asymptotic stability, 3-dimensional geometry and topology, hyperbolic, nilgeometry.

The author was supported by the Japan Society for the Promotion of Science (JSPS). The author is grateful to Vincent Moncrief and Katsuhito Yasuno for collaborations related to this work and helpful discussions. 
This article discusses linear perturbations of two Bianchi type solutions; one is Bianchi III, the other is Bianchi II. The spatial manifold of each solution is assumed to be closed (meaning 'compact without boundary'), introducing an appropriate compactification. (As a result, the solution becomes locally homogeneous.) One of the common features of these two models is that both of the spatial manifolds belong to so-called Yamabe type -1 , which is of special interest in relativity [FM]. One of the biggest differences is that while Bianchi III belongs to so-called class B, Bianchi II belongs to class A. (The class A or B is defined with respect to whether the trace of the structure constant of a Bianchi group vanishes or not. [EM]) In fact, we will see some of the largest differences between Bianchi III and II amount to those between class A and B. Therefore, in particular, the basic methods for Bianchi II will be applicable to other class A Bianchi models, too. (Difficulties in Bianchi II will also be common for all Bianchi A models.)

We deal with the Bianchi III perturbations first. In this case, we have almost complete results. The technical details of fundamental analysis of Bianchi III vacuum perturbations, including the derivation of the wave equations for the gauge-invariant variables, have already been given in [TMY] and is also briefly summarized in subsections 2.1 to 2.3 of the present article. The asymptotic stability of the perturbations has been discussed in $\mathbf{Y T}]$ in a framework of dynamical system approach, where one does not have to appeal to an exact background solution. In the present article we discuss the asymptotic stability from a different point of view in subsections 2.4 and 2.5 using the exact background solution. The issue of stability of the Bianchi II solution is on the other hand still an open question. In this article we discuss properties of some simpler field equations on that background in the subsequent section, stressing differences from the Bianchi III background.

\section{Bianchi III vacuum perturbations}

2.1. The background solution. First, let us describe the background geometry briefly.

The Bianchi III algebra is generated by three vectors $\xi_{I}(I=1 \sim 3)$ such that the commutation relation is given by

$$
\left[\xi_{1}, \xi_{2}\right]=\xi_{1}, \quad\left[\xi_{2}, \xi_{3}\right]=\left[\xi_{3}, \xi_{1}\right]=0 .
$$

Using coordinates, they can be represented as

$$
\xi_{1}=\frac{\partial}{\partial x}, \quad \xi_{2}=x \frac{\partial}{\partial x}+y \frac{\partial}{\partial y}, \quad \xi_{3}=\frac{\partial}{\partial z},
$$

We will be able to think of these vectors as Killing vectors on our background spacetime. One can also consider invariant vectors $\chi_{I}$ and 1 -forms $\sigma^{I}$ defined by

$$
\mathcal{L}_{\xi_{I}} \chi_{J}=0, \text { and } \mathcal{L}_{\xi_{I}} \sigma^{J}=0,
$$

where $\mathcal{L}_{\xi_{I}}$ denotes the Lie derivative with respect to the vector $\xi_{I}$. Suppose $\chi_{I}$ and $\sigma^{I}$ are dual to each other; $\left\langle\chi_{I}, \sigma^{J}\right\rangle=\delta_{I}^{J}$. Then, using the coordinates they are represented as

$$
\begin{gathered}
\chi_{1}=y \frac{\partial}{\partial x}, \quad \chi_{2}=y \frac{\partial}{\partial y}, \quad \chi_{3}=\frac{\partial}{\partial z} \\
\sigma^{1}=\frac{d x}{y}, \quad \sigma^{2}=\frac{d y}{y}, \quad \sigma^{3}=d z .
\end{gathered}
$$


For convenience we define a standard 2-dimensional hyperbolic metric

$$
\tilde{h} \equiv \sigma^{1} \otimes \sigma^{1}+\sigma^{2} \otimes \sigma^{2},
$$

and a standard 1-dimensional Euclid metric

$$
\tilde{l} \equiv \sigma^{3} \otimes \sigma^{3} .
$$

Then, the (universal covering) vacuum solution we consider is given by

$$
\tilde{\mathrm{g}}=-N(t)^{2} d t^{2}+q_{1}(t) \tilde{h}+q_{2}(t) \tilde{l},
$$

where the two scale functions $q_{1}(t)$ and $q_{2}(t)$ and the lapse function $N(t)^{2}$ are explicitly given by

$$
q_{1}(t)=(t+k)^{2}, \quad q_{2}(t)=(t-k) /(t+k), \quad N(t)^{2}=1 / q_{2}(t),
$$

where $k$ is a real parameter.

This type of solution is called locally rotationally symmetric (LRS), because, due to the hyperbolic metric $\tilde{h}$ the spatial metric $\tilde{q} \equiv q_{1} \tilde{h}+q_{2} \tilde{l}$ has a fourth Killing vector (with degenerate points). We consider only this type of metric, because otherwise one cannot compactify the spatial manifold.

We can express the compactified solution as follows:

$$
(M \times \mathbf{R}, \mathrm{g})=\left(\mathbf{R}^{4}, \tilde{\mathrm{g}}\right) / \Gamma,
$$

where the spatial manifold $M$ is supposed to be the direct product $\Sigma_{g} \times S^{1}$, where $\Sigma_{g}(g>1)$ is a higher genus surface with genus $g$ while is $S^{1}$ the circle. For convenience, we call $\Sigma_{g}$ the base and $S^{1}$ the fiber. $\Gamma \subset \operatorname{Isom}\left(\mathbf{R}^{4}, \tilde{\mathrm{g}}\right)$ is a discrete subgroup of the isometry group of $\left(\mathbf{R}^{4}, \tilde{\mathrm{g}}\right)$, which acts on $\left(\mathbf{R}^{4}, \tilde{\mathrm{g}}\right)$ from the left. We assume that $\Gamma$ is of orthogonal type[TMY], which means that (the action of) $\Gamma$ preserves the foliation by $H^{2}$ of the universal covering manifold. In this case one can write the spacetime metric $\mathrm{g}$ for the spatially compactified manifold in almost the same form as $\tilde{\mathrm{g}}$ :

$$
\mathrm{g}=-N(t)^{2} d t^{2}+q_{1}(t) h+q_{2}(t) l,
$$

where $h$ is the hyperbolic metric on $\Sigma_{g}$ induced from the universal cover metric $\tilde{q}$, while $l$ is the Euclid metric on $S^{1}$ induced from the same metric.

2.2. Eigenfunctions, vectors, and symmetric tensors. We need to find complete sets of eigenfunctions, (eigen)vectors, and (eigen)symmetric tensors for the Laplacian so that we can mode-expand the field variables on our background. Thanks to the fact that our spatial manifold has the orthogonal direct product property, we can reduce this task to those for the base and fiber. Namely, once one finds eigenfunctions, vectors, and symmetric tensors on both the fiber and the base, the remaining task is just to make appropriate products. For details, see [TMY]. Let us in the following recall some basic definitions.

The most fundamental are the eigenfunctions on the fiber and the base. Let $c_{m}$ be a real eigenfunction on the fiber, corresponding to eigenvalue $m$;

$$
\left(\chi_{3}\right)^{2} c_{m}=-m^{2} c_{m} \text {. }
$$

We call $m$ the fiber eigenvalue. ( $\chi_{3}=\partial_{z}$ is the derivative operator along the fiber.) We also define another real eigenfunction $\bar{c}_{m}$ by

$$
\chi_{3} c_{m}=-m \bar{c}_{m}, \quad \chi_{3} \bar{c}_{m}=m c_{m} .
$$


Similarly, let $\hat{S}_{\lambda}$ be a real eigenfunction on the base, corresponding to eigenvalue $\lambda$;

$$
\triangle_{h} \hat{S}_{\lambda}=-\lambda^{2} \hat{S}_{\lambda}
$$

We call $\lambda$ the base eigenvalue. $\left(\triangle_{h}\right.$ is the Laplacian with respect to the metric $h$.) The mode function on the spatial manifold $M=\Sigma_{g} \times S^{1}$ is given by the product $S_{m, \lambda} \equiv c_{m} \hat{S}_{\lambda}$

$$
\triangle_{q} S_{m, \lambda}=-\left(q_{1}^{-1} \lambda^{2}+q_{2}^{-1} m^{2}\right) S_{m, \lambda},
$$

where $q=q_{1} h+q_{2} l$ is the spatial part of the metric $g$.

The mode vectors and symmetric tensors on $(M, q)$ are categorized, according to those for the base $\left(\Sigma_{g}, h\right)$, into four kinds; the even type, the odd type, the harmonic type, and the transverse-traceless (ТT) type. The meaning of this is as follows.

The even vectors (or 1-forms) $\hat{S}_{a}$ on the base are equivalent to the exact (gradient) 1 -forms on the base, $\hat{S}_{a}=\hat{D}_{a} \hat{S}$, where $\hat{D}_{a}$ is the covariant derivative operator on the base. $\hat{S}$ is generally a function on the base, but it is supposed to be the mode function $\hat{S}=\hat{S}_{\lambda}$ when thinking of $\hat{S}_{a}$ as a mode vector on the base. Similarly, the odd 1-forms $\hat{V}_{a}$ are equivalent to the (Hodge-)dual exact 1-forms, $\hat{V}_{a}=\varepsilon_{a}{ }^{b} \hat{D}_{b} \hat{S}$, where $\varepsilon_{a b}=2 \sigma^{1}{ }_{[a} \sigma_{b]}^{2}$ is the area 2 -form on the base. (To raise indices, use $h^{a b}$, the inverse of $h_{a b}$.) The harmonic vectors $\hat{U}_{a}$ are the Hodge harmonic vectors defined by $d \hat{U}=\delta \hat{U}=0$, or equivalently $\hat{D}_{[a} \hat{U}_{b]}=\hat{D}^{a} \hat{U}_{a}=0$. The number of the independent harmonic one-forms is equivalent to the 1st-Betti number of the surface, which is given by $2 g$ in case of the higher genus surface. The Hodge-decomposition tells us that an arbitrary 1-form on the base can be uniquely decomposed using these even, odd, and harmonic (mode) 1-forms.

The even symmetric tensors are symmetric tensors made from the even vectors or the metric $h_{a b}$ and the mode functions. (The mode functions themselves are supposed to be of even type.) We define the even trace part by $\hat{S} h_{a b}$, and the even traceless part $\hat{S}_{a b}$ as the traceless part of the gradient of $\hat{S}_{a}$. Similarly, we define the odd symmetric tensors $\hat{V}_{a b}$ and the harmonic symmetric tensors $\hat{U}_{a b}$ by the (symmetric) gradient of $\hat{V}_{a}$ and $\hat{U}_{a}$, respectively. (They are automatically traceless.) From York's decomposition $\mathbf{Y O}$, we know that it is necessary for completeness to consider the transverse-traceless (TT) tensors $\hat{W}_{a b}$, which are defined by $\hat{D}^{b} \hat{W}_{a b}=$ $h^{a b} \hat{W}_{a b}=0$. The number of independent TT tensors on the higher genus hyperbolic surface is the same as the dimension of the so-called Teichmüller space, which is equal to $6 g-6$.

The symmetric mode tensors on the spatial manifold $M$ have, as a result, nine kinds, which are denoted as $\left(E_{i}\right)_{a b}(i=1, \cdots, 9)$. Four of them are of the even kind, which are defined as $\left(E_{1}\right)_{a b}=c_{m} \hat{S} h_{a b},\left(E_{2}\right)_{a b}=c_{m} \hat{S}_{a b},\left(E_{3}\right)_{a b}=c_{m} \hat{S} l_{a b}$, and $\left(E_{4}\right)_{a b}=2 \bar{c}_{m} \hat{S}_{(a} \sigma_{b)}^{3}$. (We omit the subscript $\lambda$ like $\hat{S}=\hat{S}_{\lambda}$.) Similarly, the odd symmetric mode tensors are defined as $\left(E_{5}\right)_{a b}=c_{m} \hat{V}_{a b}$, and $\left(E_{6}\right)_{a b}=$ $2 \bar{c}_{m} \hat{V}_{(a} \sigma_{b)}^{3}$. The harmonic symmetric mode tensors are defined as $\left(E_{7}\right)_{a b}=c_{m} \hat{U}_{a b}$, and $\left(E_{8}\right)_{a b}=2 \bar{c}_{m} \hat{U}_{(a} \sigma_{b)}^{3}$. Finally, the TT symmetric mode tensors are defined as $\left(E_{9}\right)_{a b}=c_{m} \hat{W}_{a b}$. These are orthogonal to each other with respect to the standard $L^{2}$-norm. (The mode vectors on $M$ are also defined in a similar way.) 
2.3. Vacuum perturbations and wave equations. Using the symmetric mode tensors defined above, we can expand the first variation $\delta q_{a b}$ of the spatial metric $q_{a b}$ as follows.

$$
\delta q_{a b}=\sum \gamma^{i}\left(E_{i}\right)_{a b}
$$

where the sum is taken over $i=1, \cdots, 9$, as well as all possible eigenvalues $\lambda$ and $m$. The coefficients $\gamma^{i}=\gamma^{i}(t)$ are functions of time that are supposed to be perturbation variables.

An important fundamental result that can be confirmed by direct computations is about how the perturbations decouple, which is stated as follows. For given base eigenvalue $\lambda$ and the fiber eigenvalue $m$, the set of the even variables, the odd set, the harmonic set and TT set evolve independently from each other. Note that this statement says two kinds of decoupling; first of all, different eigenmodes decouple from each other, and the four kinds of set of variables decouple from each other. Due to this decoupling property, we can deal with the perturbations one by one for each one of the four kinds for each mode.

The perturbation variables change their form by an infinitesimal diffeomorphism acting on the manifold. Certain combinations of the variables however remain invariant. Such a combination is known as the gauge invariant variable. In general, one of the central issues in perturbation analysis is to find these gauge invariant variables and determine the dynamics of them. Our choice of the gaugeinvariant variables is as follows:

$$
\begin{aligned}
& Q_{\mathrm{E}}=-\Delta_{1} \gamma^{1}-\Delta_{2} \gamma^{2}+\gamma^{3}-(2 m / \lambda) \gamma^{4}, \\
& Q_{\mathrm{O}}=(\nu / \sqrt{2}) \gamma^{5}+\gamma^{6}, \\
& Q_{\mathrm{H}}=(m / 2) \gamma^{7}+\gamma^{8}, \\
& Q_{\mathrm{T}}=\gamma^{9},
\end{aligned}
$$

where $\Delta_{1} \equiv \dot{q}_{2} / \dot{q}_{1}, \Delta_{2} \equiv\left(\lambda / \sqrt{2\left(\lambda^{2}+2\right)}\right)\left(\Delta_{1}+2 m^{2} / \lambda^{2}\right)$, and $\nu \equiv m / \sqrt{\lambda^{2}+2}$. The subscripts $\mathrm{E}, \mathrm{O}, \mathrm{H}$, and $\mathrm{T}$ are attached to express it is for the even, odd, harmonic or TT perturbation, respectively.

Getting wave equations (equivalent to the linearized Einstein equation) is a substantial part of the work in the study of perturbations. While we show only the results of it in the following, it is worth mentioning that the actual computations were done in the Hamiltonian formalism, using so-called the method of generating function to find a desired canonical transformation that is necessary to split the system into gauge-dependent and independent parts. After lengthy computations [TMY], we get the wave equations for the gauge invariant variables, which are second order ODEs of the form

$$
\ddot{Q}_{\mathrm{A}}+f_{\mathrm{A}}(t) \dot{Q}_{\mathrm{A}}+g_{\mathrm{A}}(t) Q_{\mathrm{A}}=0 . \quad(\mathrm{A}=\mathrm{E}, \mathrm{O}, \mathrm{H}, \text { or } \mathrm{T})
$$

Each coefficient function $f_{\mathrm{A}}(t)$ or $g_{\mathrm{A}}(t)$ are given as follows:

(i) For the even perturbations:

$$
f_{\mathrm{E}}=-2\left(\frac{(t-2 k)}{t_{+} t_{-}}-\frac{X}{Z}\right), \quad g_{\mathrm{E}}=m^{2} \frac{t_{+}^{2}}{t_{-}^{2}}+\lambda^{2} \frac{1}{t_{+} t_{-}}+\frac{Y}{Z},
$$


where

$$
\begin{aligned}
X & \equiv 8 m^{2}(t-2 k) t_{+}{ }^{2}+2 \lambda^{2} m^{2}(2 t-k) t_{+}{ }^{2}+2 \lambda^{2}(2 t-3 k)+\lambda^{4}(2 t-k) t / t_{+}, \\
Y & \equiv 16 m^{4} t_{+}{ }^{4}-4 k m^{2} t_{+}\left[\lambda^{2}(t-4 k) / t_{-}-8\right]+2 k^{2} \lambda^{4}(2 t+k) /\left(t_{-} t_{+}^{2}\right), \\
Z & \equiv 4 m^{4} t_{+}{ }^{6}+8 m^{2} t_{-} t_{+}{ }^{3}+4 \lambda^{2} m^{2} t t_{+}{ }^{3}+2 \lambda^{2} t_{-} t_{+}+\lambda^{4} t^{2} .
\end{aligned}
$$

(ii) For the odd and harmonic perturbations $(\lambda \neq 0$ for the odd, $\lambda=0$ for the harmonic)

$$
f_{\mathrm{O}}=-\frac{2 \nu^{2}(t-2 k) t_{+}}{t_{-} u}, \quad g_{\mathrm{O}}=\left(\lambda^{2}+2\right) \frac{u}{t_{-}^{2}}+\frac{2(t-3 k)}{t_{+}^{2} t_{-}}-\frac{4(t-2 k)}{t_{+}^{3} u},
$$

where $u \equiv \nu^{2} t_{+}^{2}+t_{-} t_{+}^{-1}$.

(iii) For the TT perturbations

$$
f_{\mathrm{T}}=-\frac{2(t-2 k)}{t_{+} t_{-}}, \quad g_{\mathrm{T}}=m^{2} \frac{t_{+}^{2}}{t_{-}^{2}}+\frac{2(t-3 k)}{t_{+}^{2} t_{-}} .
$$

2.4. Asymptotic solutions. Let us find future asymptotic solutions to discuss the future stability. A lot is known about how we can find asymptotic solutions for linear ODEs. The following simple result, taken from standard texts, is sufficient for our purpose.

TheOREm 2.1 (e.g., $[\mathbf{C H}]$ ). Consider the following second order ODE

$$
X^{\prime \prime}+f(s) X=0,
$$

where $f(s)$ is a function of $s$ that approaches a constant

$$
\lim _{s \rightarrow \infty} f(s)=C=\text { constant. }
$$

(Primes' stand for $d / d s$.) If $C \neq 0$ and

$$
\int^{\infty}|f(s)-C| d s<\infty
$$

then the equation (2.21) has a set of fundamental solutions $\left\{e^{ \pm i \sqrt{C} s}(1+o(1))\right\}$ when $C>0$, or $\left\{e^{ \pm \sqrt{|C|} s}(1+o(1))\right\}$ when $C<0$. If $C=0$ and

$$
\int^{\infty} s|f(s)| d s<\infty
$$

then the equation (2.21) has a set of fundamental solutions $\{1+o(1), s(1+o(1))\}$.

With the aid of this theorem we can prove the following.

Theorem 2.2 (Asymptotic solutions for the generic case). Assume that the fiber eigenvalue $m$ does not vanish, $m \neq 0$. Then, the wave equations 2.17) for the gauge-invariant variables (2.16) possess the following fundamental solutions

$$
\begin{aligned}
& Q_{\mathrm{A}}(t)=\left\{t e^{ \pm i(m t+2 k \log t)}(1+o(1))\right\} . \\
& (\mathrm{A}=\mathrm{E}, \mathrm{O}, \mathrm{H}, \text { or } \mathrm{T})
\end{aligned}
$$


Proof. Define a new time coordinate $s$ by

$$
\frac{d s}{d t}=\sigma(t) \equiv 1+\frac{2 k}{t}+O\left(\frac{1}{t^{2}}\right) .
$$

Rewrite the wave equation (2.17) in terms of $s$. Then, by taking

$$
a_{\mathrm{A}}=\frac{1}{\sqrt{\sigma}} e^{-\frac{1}{2} \int f_{\mathrm{A}} d t}
$$

and putting $Q_{\mathrm{A}}=a_{\mathrm{A}} X_{\mathrm{A}}$, one has an alternative equation $X_{\mathrm{A}}^{\prime \prime}+W_{\mathrm{A}}(s) X_{\mathrm{A}}=0$. It is easy to check that $\int^{\infty}\left|W_{\mathrm{A}}(s)-m^{2}\right| d s<\infty$. Therefore, from the previous theorem, $X_{A}$ has the fundamental solutions $e^{ \pm i m s}(1+o(1))$, from which the claim follows.

Similarly, we can prove the following for the $m=0$ case. This case is called the $U(1)$-symmetric case, because this kind of perturbation keeps the $U(1)$-symmetry of the background along the $S^{1}$ fibers.

TheOREM 2.3 (Asymptotic solutions for the $U(1)$-symmetric case). Assume $m=0$. Define $\rho_{\lambda}=\lambda^{2}-1 / 4$, for convenience. Then, the wave equations [2.17) for the gauge-invariant variables [2.16) possess the following fundamental solutions:

For the even perturbations:

$$
Q_{\mathrm{E}}(t)= \begin{cases}\left\{\frac{1}{\sqrt{t}} e^{ \pm i \sqrt{\rho_{\lambda}} \log t}(1+o(1))\right\} & \left(\lambda^{2}>\frac{1}{4}\right) \\ \left.\frac{1}{\sqrt{t}}(1+o(1)), \frac{\log t}{\sqrt{t}}(1+o(1))\right\} & \left(\lambda^{2}=\frac{1}{4}\right) \\ \left.t^{-\frac{1}{2} \pm \sqrt{\left|\rho_{\lambda}\right|}}(1+o(1))\right\} . & \left(\lambda^{2}<\frac{1}{4}\right)\end{cases}
$$

For the odd perturbations:

$$
Q_{\mathrm{O}}(t)= \begin{cases}\left\{\sqrt{t} e^{ \pm i \sqrt{\rho_{\lambda}} \log t}(1+o(1))\right\} & \left(\lambda^{2}>\frac{1}{4}\right) \\ \{\sqrt{t}(1+o(1)), \sqrt{t} \log t(1+o(1))\} & \left(\lambda^{2}=\frac{1}{4}\right) \\ \left\{t^{\frac{1}{2} \pm \sqrt{\left|\rho_{\lambda}\right|}}(1+o(1))\right\} . & \left(\lambda^{2}<\frac{1}{4}\right)\end{cases}
$$

Proof. Define a new time coordinate $s$ by

$$
\frac{d s}{d t}=\sigma^{(0)}(t) \equiv \frac{1}{t}+O\left(\frac{1}{t^{2}}\right) .
$$

Rewrite the wave equation (2.17) in terms of $s$. Then, by taking

$$
a_{A}=\frac{1}{\sqrt{\sigma^{(0)}}} e^{-\frac{1}{2} \int f_{\mathrm{A}} d t}
$$

and putting $Q_{\mathrm{A}}=a_{A} X_{\mathrm{A}}$, one has an alternative equation $X_{\mathrm{A}}^{\prime \prime}+W_{\mathrm{A}}(s) X_{\mathrm{A}}=0$. $(\mathrm{A}=\mathrm{E}$ or $\mathrm{O}$.) It is straightforward to check that

$$
\int^{\infty}\left|W_{A}(s)-\rho_{\lambda}\right| d s<\infty \quad\left(\lambda^{2} \neq \frac{1}{4}\right)
$$

and

$$
\int^{\infty} s\left|W_{A}(s)\right| d s<\infty . \quad\left(\lambda^{2}=\frac{1}{4}\right)
$$

Theorem 2.1 therefore applies, and the claim follows after replacing the time coordinate back to $t$. 
REMARK 2.4. As seen from the last two proofs, the key point to find asymptotic solutions is the choice of a good new time variable (like the choice of $\sigma(t)$ or $\sigma^{(0)}(t)$ in the proofs.).

Interestingly enough, in the generic case the asymptotic solutions are common for all kinds of perturbations. Moreover, the asymptotic solution does not depend on the base eigenvalue $\lambda$. In this sense the asymptotic solutions are very universal.

On the other hand, for the $U(1)$-symmetric case, for each kind of perturbations the behavior of the asymptotic solutions is divided into three cases, depending upon the value of $\lambda$.

2.5. Stability issue. Although all the asymptotic solutions above show that our gauge-invariant perturbation variables are growing in time, one cannot say anything about stability of the solution from these results themselves. This is because they do not take into account the fact that the background solution is expanding. Because of the anisotropy of the background solution, it is a very subtle question how one can subtract this expansion effect. If the background was isotropic there exists a natural way to normalize the perturbation variables, because it has only one scale factor, but in an anisotropic case it is not clear how to find a right way of normalizing the variables, especially in a way such that the result is gauge invariant.

Nevertheless, one possible way may be to use the zero mode solution to normalize the gauge invariant variables. By a zero mode solution we mean a solution with the vanishing eigenvalues. Such a solution represents a perturbation from a locally homogeneous solution to another locally homogeneous solution, which is an effect we are not interested in. So, it may make sense to use the zero mode solution to do a normalization. Moreover, it is apparently gauge-invariant. (This scheme is essentially equivalent to the one discussed in $[\mathbf{Y T}]$.)

All the zero mode (vacuum) solutions are already given in [TMY], from which one obtains the following growing rates for those solutions:

$$
Q_{\mathrm{E}}^{(0)}=O(1), \quad Q_{\mathrm{H}}^{(0)}=O(t), \quad Q_{\mathrm{T}}^{(0)}=O\left(t^{2}\right) .
$$

(Although there does not exist a zero mode for the odd perturbations, we identify it with that of the harmonic ones, recalling the fact that the harmonic perturbation system is formally equivalent to the limit $\lambda \rightarrow 0$ of the odd system.)

We define the stability measures by means of zero mode normalization by

$$
M_{\mathrm{A}} \equiv Q_{\mathrm{A}} / Q_{\mathrm{A}}^{(0)}, \quad(\mathrm{A}=\mathrm{E}, \mathrm{O}, \mathrm{H}, \text { or } \mathrm{T})
$$

where we think of $Q_{\mathrm{O}}^{(0)}=Q_{\mathrm{H}}^{(0)}$ as noted above. From the asymptotic solutions for $Q_{\mathrm{A}}$ we immediately obtain the following behaviors for our stability measures.

THEOREM 2.5. The stability measures by means of zero mode normalization for the vacuum orthogonal Bianchi III spacetime have the following asymptotic decaying or growing rates:

Generic $(m \neq 0)$ case:

$$
\left|M_{\mathrm{A}}\right|= \begin{cases}O(t) & (A=E), \\ O(1) & (A=O \text { or } H) \\ O\left(t^{-1}\right) & (A=T) .\end{cases}
$$


(The rates are common for all eigenvalues (except $m=0$ ), but depend on the kind of perturbations.)

$U(1)$-symmetric $(m=0)$ case:

$$
\left|M_{\mathrm{A}}\right|= \begin{cases}O\left(t^{-1 / 2}\right) & \left(\lambda^{2}>\frac{1}{4}\right) \\ O\left(\frac{\log t}{\sqrt{t}}\right) & \left(\lambda^{2}=\frac{1}{4}\right) \\ O\left(t^{-1 / 2+\sqrt{\left|\rho_{\lambda}\right|}}\right) & \left(\lambda^{2}<\frac{1}{4}\right)\end{cases}
$$

for $A=E$ or $O$. (The rates are common for the even and odd kinds, but there exists a critical eigenvalue $\lambda^{2}=1 / 4$ for each kind.)

REMARK 2.6. One can replace (without any modifications) the time variable $t$ to the proper time $\tau$ in the rate formulas of the above theorem.

Note that we have three kinds of behaviors for the generic case; The TT perturbations are decaying, so we can say that the solution is stable against this kind of perturbations. The odd and harmonic perturbations approach a constant, so in this case the solution is quasi-stable. Finally, the even perturbations are growing, so the solution is unstable against the even perturbations.

On the other hand all the $U(1)$-symmetric perturbations are decaying, so we can say that the solution is stable against the $U(1)$-symmetric perturbations. Although this means that the $U(1)$-symmetric perturbations play no major role in the whole perturbations of our system, the existence of the critical base eigenvalue $\lambda^{2}=1 / 4$ is worth pointing out. A similar existence of critical value has also been reported in a nonlinear (but $U(1)$-symmetric) analysis [CBM].

\section{Field equations on a closed Bianchi II solution}

3.1. The background solution. The Bianchi II (or Nil) group $G_{\mathrm{II}}$ is the 3 -dimensional Lie group which consists of all real upper triangular matrices of the form $\left(\begin{array}{lll}1 & x & z \\ 0 & 1 & y \\ 0 & 0 & 1\end{array}\right)$ with the usual multiplication rule for matrices. To save space let us write an element $\mathbf{x} \in G_{\mathrm{II}}$ in (transposed) vector form $\mathbf{x}=(x, y, z)$. Then the multiplication rule is given by $(a, b, c) \circ(x, y, z)=(a+x, b+y, c+z+a y)$ for another $\mathbf{a}=(a, b, c) \in G_{\mathrm{II}}$. The group $G_{\mathrm{II}}$ acts on our universal covering spatial

manifold $\tilde{M}=\mathbf{R}^{3}$ from the left, identifying the group manifold $G_{\mathrm{II}}$ with $\tilde{M}$. The Bianchi II algebra is generated by the following three vectors $\xi_{I}(I=1 \sim 3)$

$$
\xi_{1}=\frac{\partial}{\partial x}+y \frac{\partial}{\partial z}, \quad \xi_{2}=\frac{\partial}{\partial y}, \quad \xi_{3}=\frac{\partial}{\partial z}
$$

which serve as Killing vectors on our background space or spacetime. The invariant vectors $\chi_{I}$ and their dual 1 -forms $\sigma^{I}$ are given by

$$
\begin{gathered}
\chi_{1}=\frac{\partial}{\partial x}, \quad \chi_{2}=\frac{\partial}{\partial y}+x \frac{\partial}{\partial z}, \quad \chi_{3}=\frac{\partial}{\partial z}, \\
\sigma^{1}=d x, \quad \sigma^{2}=d y, \quad \sigma^{3}=d z-x d y .
\end{gathered}
$$

Like the Bianchi type III solution the Bianchi type II vacuum solution can be expressed as

$$
\left(M \times \mathbf{R}, \mathrm{g}_{a b}\right)=\left(\tilde{M} \times \mathbf{R}, \tilde{\mathrm{g}}_{a b}\right) / \Gamma
$$


using the universal cover solution $\left(\tilde{M} \times \mathbf{R}, \tilde{g}_{a b}\right)$ and a covering map $\Gamma$ acting on it. The spatial manifold $M$ here is, for definiteness, specified to be the "circle bundle over the torus $\left(T^{2}\right)$ with Euler number $e=1$." (See, e.g., [HS.) The fundamental group can be represented in the standard notation as

$$
\pi_{1}(M)=\left\langle g_{1}, g_{2}, g_{3} ;\left[g_{1}, g_{2}\right]=g_{3},\left[g_{1}, g_{3}\right]=1,\left[g_{2}, g_{3}\right]=1\right\rangle,
$$

where the brackets stand for commutators, $[a, b] \equiv a b a^{-1} b^{-1}$. The vacuum metric $\tilde{\mathrm{g}}_{a b}$ on the universal cover is given below. $\Gamma \subset G_{\mathrm{II}}$ is a discrete subgroup of the Bianchi II group $G_{\mathrm{II}}$, which acts on the universal covering spacetime $\left(\tilde{M} \times \mathbf{R}, \tilde{\mathrm{g}}_{a b}\right)$ from the left in the way that keeps the natural homogeneous slicings. Some details for the compactification are given in the next subsection. The metric $\mathrm{g}_{a b}$ on $M \times \mathbf{R}$ is the induced metric from $\tilde{\mathrm{g}}_{a b}$.

The exact vacuum solution $\tilde{\mathrm{g}}_{a b}$ is given [TAU] by

$$
d s^{2}=-N^{2}(t) d t^{2}+q_{1}(t)\left(\sigma^{1}\right)^{2}+q_{2}(t)\left(\sigma^{2}\right)^{2}+q_{3}(t)\left(\sigma^{3}\right)^{2},
$$

where

$$
N^{2}=1+\beta^{2} t^{4 p_{3}}, \quad q_{1}=t^{2 p_{1}} N^{2}, \quad q_{2}=t^{2 p_{2}} N^{2}, \quad q_{3}=16 p_{3}^{2} \beta^{2} t^{2 p_{3}} / N^{2} .
$$

$p_{i}(i=1,2,3)$ and $\beta$ are constant parameters such that $\beta>0, p_{3} \neq 0$, and

$$
\Sigma p_{i}=\Sigma p_{i}^{2}=1 \text {. }
$$

When $p_{1}=p_{2}$, or equivalently when $q_{1}(t)=q_{2}(t)$, the solution is said to be LRS as in the Bianchi III case. While there seem two possible such cases $\left(p_{1}, p_{2}, p_{3}\right)=$ $(0,0,1)$ and $(2 / 3,2 / 3,-1 / 3)$, these two solutions represent the same one-parameter solution. When we consider a LRS solution, we may want to take $\left(p_{1}, p_{2}, p_{3}\right)=$ $(2 / 3,2 / 3,-1 / 3)$, since the time coordinate $t$ in this solution approaches the proper time at future infinity, which is more favorable for comparisons with other models.

As shown in [KKH] $\Gamma$ is a four-parameter group. Our spatially closed solution (3.3) therefore comprises a six parameter solution (since the universal cover has, as we have seen, two independent parameters).

Notice the fact that $\Gamma$ is a subgroup of the Bianchi II group $G_{\text {II }}$, not necessarily a subgroup of a larger group like in the Bianchi III case. This in particular means that each invariant vector $\chi_{I}$ is well defined not only on the universal cover $\tilde{M}$ but also on the compactified manifold $M$ (i.e., the induced vectors $\pi^{*} \chi_{I}$ for the covering map $\pi: \tilde{M} \rightarrow M$ are well defined on $M$. We omit $\pi^{*}$ for simplicity, though). This is because they are invariant under the action of $G_{\mathrm{II}}$, and so are under $\Gamma \subset G_{\mathrm{II}}$. We will see that because of this property the invariant vectors $\chi_{I}$ play a central role in developing calculus concerning mode expansions. This significance of the invariant vectors is common for all Bianchi class A models.

3.2. Compactifications and eigenfunctions. Consider an arbitrary locally homogeneous closed 3-manifold $\left(M, q_{a b}\right)$ with $\pi_{1}(M)$ given by (3.4). Such a Riemannian manifold is isometric to the quotient $\mathcal{N}^{3} / A$, where $\mathcal{N}^{3}=\left(\mathbf{R}^{3}, e^{2 \alpha} q_{a b}^{(0)}\right)$ is the universal cover with a metric that is conformal to the Bianchi II standard metric $q^{(0)}=\left(\sigma^{1}\right)^{2}+\left(\sigma^{2}\right)^{2}+\left(\sigma^{3}\right)^{2} \cdot e^{2 \alpha}$ is a constant scale factor. $A \subset \operatorname{Isom} \mathcal{N}^{3}$ is a three parameter infinite group generated by three generators $\mathbf{a}_{i} \in G_{\mathrm{II}} \subset \operatorname{Isom} \mathcal{N}^{3}$ $(i=1 \sim 3)$, which are of the form (cf. $[\mathbf{K T H}])$

$$
\mathbf{a}_{1}=(u, \delta, 0), \quad \mathbf{a}_{2}=(0,2 \pi v, 0), \quad \mathbf{a}_{3}=(0,0,2 \pi u v) .
$$


We denote $A=\left\{\mathbf{a}_{1}, \mathbf{a}_{2}, \mathbf{a}_{3}\right\}$. The three parameters $u, v$, and $\delta$ are called the Teichmüller parameters.

Also, consider another Riemannian manifold $\left(\bar{M}, \bar{q}_{a b}\right)$, which can be represented as $\mathcal{N}^{3} / \bar{A}$, where $\bar{A}=\left\{\mathbf{a}_{2}, \mathbf{a}_{3}\right\}$. $\left(\bar{M}, \bar{q}_{a b}\right)$ is a covering of $\left(M, q_{a b}\right) . \bar{M}$ is homeomorphic to the direct product $T^{2} \times \mathbf{R}$, where $T^{2}$ is the two-torus.

Note that the (scalar) Laplacian $\triangle$ with respect to the standard metric $q_{a b}^{(0)}$ can be expressed as $\Delta=\left(\chi_{1}\right)^{2}+\left(\chi_{2}\right)^{2}+\left(\chi_{3}\right)^{2}$. This operator is apparently well defined on both $\bar{M}$ and $M$, since so is each $\chi_{I}$. It is straightforward to confirm that $\triangle$ commutes with $\chi_{3}$. We can therefore diagonalize eigenfunctions with respect to both $\triangle$ and $\chi_{3}$. Consider another operator $\xi_{2}=\partial / \partial y$, which we can find commutes with both $\triangle$ and $\chi_{3}$, so one can diagonalize the eigenfunctions with respect to $\xi_{2}$, too. This operator however is not well defined on $M$, but on $\bar{M}$. Because of this fact, it is convenient to first consider eigenfunctions on $\bar{M}$, and then construct those on $M$, taking linear combinations of the eigenfunctions on $\bar{M}$.

Let us define eigenvalues $\mu$ and $\nu$ for the operators $\chi_{3}$ and $\xi_{2}$ by the following relations

$$
\chi_{3} \bar{\phi}=i \mu \bar{\phi}, \quad \xi_{2} \bar{\phi}=i \nu \bar{\phi}
$$

where $\bar{\phi}$ is an eigenfunction on $\bar{M}$. Also, we define $\lambda$ by $\triangle \bar{\phi}=-\lambda^{2} \bar{\phi}$. From the boundary condition $\bar{\phi}(\bar{\Gamma} \mathbf{x})=\bar{\phi}(\mathbf{x})$, it is found $\bar{\phi}=X(x) e^{i \mu z} e^{i \nu y}$, where

$$
\begin{aligned}
& \mu=m /(u v), \quad m=0, \pm 1, \cdots, \pm \infty, \\
& \nu=n / v, \quad n=0, \pm 1, \cdots, \pm \infty .
\end{aligned}
$$

We call $m$ (or $\mu$ ) the fiber eigenvalue, $n$ (or $\nu$ ) the auxiliary eigenvalue, $\lambda$ the total eigenvalue. The function $X(x)$ must satisfy the following equation (that is equivalent to the harmonic oscillator Schrödinger equation):

$$
X^{\prime \prime}+\left(\lambda^{2}-\mu^{2}-(\mu x+\nu)^{2}\right) X=0 .
$$

This equation tells us in particular that like the Bianchi III case, one must deal with the generic $(m \neq 0)$ case and the $U(1)$-symmetric $(m=0)$ case separately.

Let us focus on the generic $(m \neq 0)$ case. One can define the eigenfunctions on $\bar{M}$ as

$$
\bar{\phi}_{l, m, n}(\mathbf{x})=D_{l}\left( \pm \sqrt{\frac{2 u v}{|m|}}\left(\frac{m}{u v} x+\frac{n}{v}\right)\right) e^{i \frac{m}{u v} z} e^{i \frac{n}{v} y},
$$

where $D_{l}(\zeta)=e^{-\frac{1}{4} \zeta^{2}} H_{l}(\zeta)$ is the parabolic cylinder function defined using the Hermite function $H_{l}(\zeta)=(-1)^{l} e^{\frac{1}{2} \zeta^{2}} \frac{d^{l}}{d \zeta^{l}} e^{-\frac{1}{2} \zeta^{2}}$. The $D_{l}$ factor in the above equation satisfies Eq. (3.11). The plus and minus signs, respectively, correspond to $m>0$ and $m<0$ cases. The index $l$ takes non-negative integers

$$
l=0,1, \cdots, \infty .
$$

This is related to the total eigenvalue through $\lambda^{2}=|\mu|(2 l+|\mu|+1)$.

The eigenfunctions on $M$ are, as stated, expressed as infinite sums of these eigenfunctions on $\bar{M}$.

TheOrem 3.1. Consider the generic $(m \neq 0)$ modes. Using the eigenfunctions $\bar{\phi}_{l, m, n}(\mathbf{x})$ on the covering $\left(\bar{M}=T^{2} \times \mathbf{R}, \bar{q}_{a b}\right)$, the eigenfunctions on the closed 
manifold $\left(M, q_{a b}\right)$ are represented as the infinite sum

$$
\phi_{l, m, n_{0}}(\mathbf{x})=\sum_{k=-\infty}^{\infty} e^{i \delta\left(n_{0} k+m \frac{k(k-1)}{2}\right)} \bar{\phi}_{l, m, n_{0}+m k}(\mathbf{x}),
$$

where $l=0,1, \cdots, \infty, \quad|m|=1,2, \cdots, \infty, \quad n_{0}=0,1, \cdots,|m|-1$.

Proof. Since the summand is well defined on $\bar{M}$, which means it is invariant under the action of $\mathbf{a}_{2}$ and $\mathbf{a}_{3}$, all one has to confirm is the invariance of the left hand side under the action of $\mathbf{a}_{1}$. It is easy to see the following transformation rule

$$
\begin{aligned}
\bar{\phi}_{l, m, n}\left(\mathbf{a}_{1} \circ \mathbf{x}\right) & =D_{l}\left( \pm \sqrt{\frac{2 u v}{|m|}}\left(\frac{m}{u v}(x+u)+\frac{n}{v}\right)\right) e^{i \frac{m}{u v}(z+u y)} e^{i \frac{n}{v}(y+\delta)} \\
& =e^{i \frac{n}{v} \delta} D_{l}\left( \pm \sqrt{\frac{2 u v}{|m|}}\left(\frac{m}{u v} x+\frac{m+n}{v}\right)\right) e^{i \frac{m}{u v} z} e^{i \frac{m+n}{v} y} \\
& =e^{i \frac{\delta}{v} n} \bar{\phi}_{l, m, n+m}(\mathbf{x}) .
\end{aligned}
$$

From this, one can confirm the invariance

$$
\begin{aligned}
\phi_{l, m, n_{0}}\left(\mathbf{a}_{1} \circ \mathbf{x}\right) & =\sum_{k=-\infty}^{\infty} e^{i \frac{\delta}{v}\left(n_{0} k+m \frac{k(k-1)}{2}\right)} e^{i \frac{\delta}{v}\left(n_{0}+m k\right)} \bar{\phi}_{l, m, n_{0}+m(k+1)}(\mathbf{x}) \\
& =\sum_{k=-\infty}^{\infty} e^{i \frac{\delta}{v}\left(n_{0}(k+1)+m \frac{k(k+1)}{2}\right)} \bar{\phi}_{l, m, n_{0}+m(k+1)}(\mathbf{x}) \\
& =\phi_{l, m, n_{0}}(\mathbf{x}) .
\end{aligned}
$$

(The last equality follows from the replacement $k \rightarrow k-1$.)

Note that as a result of the complete compactification, the index $n_{0}$ for the eigenfunction on $M$ is bounded by $m$.

These eigenfunctions have the following properties.

TheOREM 3.2. For the eigenfunctions for the generic $(m \neq 0)$ modes, the following is fulfilled (indices $m$ and $n_{0}$ are for simplicity suppressed):

$$
\begin{aligned}
\chi_{1} \phi_{l} & =-\sqrt{\frac{|\mu|}{2}}\left[\phi_{l+1}-l \phi_{l-1}\right], \\
\chi_{2} \phi_{l} & = \pm i \sqrt{\frac{|\mu|}{2}}\left[\phi_{l+1}+l \phi_{l-1}\right], \\
\chi_{3} \phi_{l} & =i \mu \phi_{l},
\end{aligned}
$$

where $\mu$ is defined in Eq. (3.10) and the plus and minus signs correspond, respectively, to $m>0$ and $m<0$.

Proof. Use $D_{l}^{\prime}(\zeta)=-\frac{1}{2}\left(D_{l+1}(\zeta)-l D_{l-1}(\zeta)\right)$. The remaining task is a straightforward computation.

REMARK 3.3. These relations can also be derived in a purely algebraic manner. ${ }^{1}$

\footnotetext{
${ }^{1}$ In fact, defining $A_{1}=\chi_{1}+i \chi_{2}$ and $A_{2}=\chi_{1}-i \chi_{2}$, one can check commutation relations

$$
\left[\triangle, A_{1}\right]=2 i A_{1} \chi_{3}, \quad\left[\triangle, A_{2}\right]=-2 i A_{2} \chi_{3},
$$
}


3.3. Scalar field. As a direct application it is of great interest to see the scalar field equation on our Bianchi II background. For simplicity, let us consider a background solution where $\Gamma=A$, i.e., $\Gamma$ is specified by three parameters as in the form of $A$. We use the symbols $u, v$ and $\delta$ as in the $A$. These parameters are constants throughout the spacetime. (This is not to say the Teichmüller parameters for a Cauchy surface are constants of motion. TKH ) In such a case, we can use the eigenfunctions obtained in the previous section without any modifications. We expand the scalar field $\Phi$ as follows:

$$
\Phi(t, \mathbf{x})=\sum_{l, m, n_{0}} a_{l, m, n_{0}}(t) \phi_{l, m, n_{0}}(\mathbf{x}) .
$$

Since the operators $\chi_{I}$ do not change $m$ and $n_{0}$ when acting on $\phi_{l, m, n_{0}}$, we suppress these subscripts and write simply $a_{l}$ and $\phi_{l}$, as far as no confusion occurs.

The scalar field equation $0=\mathrm{g}^{a b} \nabla_{a} \nabla_{b} \Phi$ on our background reduces to

$$
0=\frac{-1}{\sqrt{-g}}\left(\sqrt{-g} N^{-2} \dot{\Phi}\right)+\left(q_{1}^{-1}\left(\chi_{1}\right)^{2}+q_{2}^{-1}\left(\chi_{2}\right)^{2}+q_{3}^{-1}\left(\chi_{3}\right)^{2}\right) \Phi
$$

where a dot stands for $d / d t$. Using the background spacetime solution for Bianchi II and the $\chi$-relations, we obtain the following wave equations:

$$
\ddot{a}_{l}+\frac{1}{t} \dot{a}_{l}+\mu f(t) a_{l}=\frac{\mu}{2}\left(t^{-2 p_{1}}-t^{-2 p_{2}}\right)\left[a_{l-2}+(l+2)(l+1) a_{l+2}\right]
$$

where

$$
f(t) \equiv \frac{2 l+1}{2}\left(t^{-2 p_{1}}+t^{-2 p_{2}}\right)+\frac{\mu}{16\left(p_{3}\right)^{2} \beta^{2}}\left(1+\beta^{2} t^{4 p_{3}}\right)^{2} t^{-2 p_{3}} .
$$

(In Eq. (3.23), $a_{l<0}$ should be considered as zero.)

A notable property of these equations is that they comprise a set of infinitely coupled equations, unless the solution is LRS. These couplings between different modes are an unavoidable feature for the field equations on a non-LRS spacetime solution, because for those backgrounds the mode functions defined with respect to a standard metric are no longer eigenfunctions with respect to the Laplacian for each spatial section. That is, those eigenfunctions defined on the standard Riemannian manifold do not remain eigenfunctions in the course of the anisotropic expansion. One could define another "time-dependent" eigenfunctions to get around this difficulty, but in that case time-derivatives of the eigenfunctions appear in the field equation, so in any case the complexities caused by anisotropic expansion are unavoidable.

The above wave equation gives a closed equation when the background is LRS. In this case we can again find asymptotic solutions:

which tell us, e.g., that

$$
\triangle A_{1} \phi=\left(\left[\triangle, A_{1}\right]+A_{1} \triangle\right) \phi=\left(2 i A_{1} \chi_{3}+A_{1} \triangle\right) \phi=-\left(2 \mu+\lambda^{2}\right) A_{1} \phi .
$$

This means that $A_{1} \phi$ is an eigenfunction for eigenvalue $\lambda^{\prime 2}=\lambda^{2}+2 \mu$, and the operator $A_{1}$ acts as an increment (decrement) operator for $m>0(m<0)$. Similarly, $A_{2}$ is a decrement (increment) operator with $\lambda^{\prime 2}=\lambda^{2}-2 \mu$ for $m>0(m<0)$. Using these relations and following a similar argument for, e.g., the $S O(3)$ case to determine the ground state, one reaches at the following relations

$$
\begin{aligned}
& A_{1} \phi_{l, m}=-\sqrt{2 \mu} \phi_{l+1, m}, \quad A_{2} \phi_{l, m}=\sqrt{2 \mu} l \phi_{l-1, m}, \quad \text { for } m>0 \\
& A_{1} \phi_{l, m}=\sqrt{2|\mu|} l \phi_{l-1, m}, \quad A_{2} \phi_{l, m}=-\sqrt{2|\mu|} \phi_{l+1, m}, \quad \text { for } m<0
\end{aligned}
$$

which are equivalent to the relations claimed. 
THEOREM 3.4. On the LRS Bianchi II vacuum solution with $p_{1}=p_{2}=2 / 3$ and $p_{3}=-1 / 3$, the scalar field equation (3.23) for the generic mode has the following fundamental solutions as $t \rightarrow \infty$ :

$$
a_{l}(t)=\left\{t^{-\frac{2}{3}} e^{ \pm i \frac{3 \mu}{4 \beta}\left(\frac{3}{4} t^{4 / 3}+\beta^{2} \log t\right)}(1+o(1))\right\} .
$$

Proof. As remarked in the previous section, it is a suitable choice of new time variable (denoted $s$ ) that is essential to have an asymptotic solution. An appropriate choice for the present equation is

$$
\frac{d s}{d t}=\frac{3}{4}\left(\frac{t^{1 / 3}}{\beta}+\frac{\beta}{t}\right) .
$$

One can show the claim following the same procedure as in THEоREм 2.2 or 2.3

3.4. Vectors and electromagnetic field. Let us consider how we can perform separation of variable for vector fields. An advantage that our Bianchi II model has and is common for other class A spatially closed Bianchi models is that in principle, we do not have to define suitable eigenvectors for this purpose. This is because there exists a set of well-defined independent three invariant vectors $\chi_{I}$ on the compactified manifold $M$. Any vectors on $M$ can be expanded with respect to these invariant vectors (i.e., one can consider the components with respect to the frame $\left.\left\{\chi_{I}\right\}\right)$ in a group invariant way and so all one has to do is to mode-expand the components using the already defined eigenfunctions $\phi_{l, m, n_{0}}$. However, this straightforward expansion is found not to be very useful for a successful analysis.

A nicer way is to use an analogy with Regge-Wheeler's spherically symmetric case or one with Bianchi III hyperbolically symmetric case, where one can take advantage of the spherically or hyperbolically symmetric planes. In those cases, one is able to define so-called the 'even' vectors and 'odd' vectors on the symmetric plane (as seen in the previous section for the Bianchi III case), and the even and odd part equations for a linear vector field equation decouple from each other.

Although there do not exist similar symmetric planes for the Bianchi II case, we can, instead, consider the "plane field" spanned by $\chi_{1}$ and $\chi_{2}$. This plane field is not integrable, because the commutator $\left[\chi_{1}, \chi_{2}\right]=\chi_{3}$ is independent from $\chi_{1}$ and $\chi_{2}$. Nevertheless, the plane field can play a special role, because the metric can be LRS only when $q_{1}=q_{2}$.

We define the area two-form $\varepsilon$ of the plane field by $\varepsilon_{a b}=2 \sigma^{1}{ }_{[a} \sigma_{b]}^{2}$. We may define the even vectors by $S_{a}=\left(\chi_{1} \phi_{l}\right) \sigma^{1}{ }_{a}+\left(\chi_{2} \phi_{l}\right) \sigma_{a}^{2}$, and odd vectors by $V_{a}=i \varepsilon_{a}^{b} \partial_{b} \phi_{l}=i\left(\left(\chi_{2} \phi_{l}\right) \sigma^{1}{ }_{a}-\left(\chi_{1} \phi_{l}\right) \sigma_{a}^{2}\right)$. (To raise an index for $\varepsilon_{a b}$ we use the standard Bianchi II metric.) We also need orthogonal vectors $S_{a}^{\perp}=\left(\chi_{3} \phi_{l}\right) \sigma^{3}{ }_{a}$ and time-like vectors $S_{a}^{\mathrm{T}}=N^{-1} \phi_{l}(d t)_{a}$, which may be considered to belong to the even part. These four kinds of vectors complete our set of eigenvectors.

Because of the fact that the commutator of $\chi_{1}$ and $\chi_{2}$ does not belong to the tangent space spanned by $\chi_{1}$ and $\chi_{2}$, the even and odd parts defined this way do not decouple from each other, even when the background is LRS. Nonetheless, the use of even and odd vectors is found useful, especially when the background is LRS, since in that case each mode is found to decouple from the others. (Do not confuse couplings between the even and odd parts and ones between modes.) When the spacetime is not LRS, however, we will have couplings between different modes due to the anisotropic expansion. 
Using these mode vectors, we can expand the vector potential for an electromagnetic field as follows:

$$
A_{a}=\sum_{l, m, n_{0}} \alpha_{0}(t) S_{a}^{\mathrm{T}}+\alpha_{1}(t) S_{a}+\alpha_{2}(t) V_{a}+\alpha_{3}(t) S_{a}^{\perp} .
$$

The four kinds of functions $\alpha_{i}(t)(i=0 \sim 3)$ serve as the field variables. Let us consider the LRS case below, on which background, as mentioned above, couplings between different modes vanish.

The quantities we are most interested in are the $(U(1)$-)gauge-invariant variables, which can be easily found by looking at components of the field strength $F_{a b}=\partial_{a} A_{b}-\partial_{b} A_{a}$. We obtain the following four independent gauge invariant variables:

$$
Q_{1}=\alpha_{1}-\alpha_{3}, Q_{2}=\alpha_{2}, P_{1}=\dot{\alpha}_{1}-\alpha_{0}, P_{2}=\dot{\alpha}_{2} .
$$

Although function $P_{3} \equiv \dot{\alpha}_{3}-\alpha_{0}$ is also gauge invariant, it is found that it can be (consistently) solved with the others, due to the constraint part of Maxwell's equation. The evolution equations for the LRS background are found as follows:

$$
\begin{aligned}
& \dot{Q}_{1}=P_{1}+\frac{1}{\mu} \frac{q_{3}}{q_{1}}\left((2 l+1) P_{1}+P_{2}\right), \quad \dot{Q}_{2}=P_{2}, \\
& \dot{P}_{1}=\left(\frac{\dot{N}}{N}-\frac{1}{2} \frac{\dot{q}_{3}}{q_{3}}\right) P_{1}-\mu^{2} \frac{N^{2}}{q_{3}} Q_{1}, \\
& \dot{P}_{2}=\left(\frac{\dot{N}}{N}-\frac{1}{2} \frac{\dot{q}_{3}}{q_{3}}\right) P_{2}-\mu^{2} \frac{N^{2}}{q_{3}} Q_{2}-\mu \frac{N^{2}}{q_{1}}\left(Q_{1}+(2 l+1) Q_{2}\right) .
\end{aligned}
$$

To tackle the gravitational perturbation equation we need to generalize our framework so that it is applicable to systems with symmetric tensors. Again, thanks to the well-defined invariant vectors $\chi_{I}$, separations of variable for the tensors are straightforward in principle. However, because of the complexity of the linearized Einstein equation, to make our analysis successful we will need to find a wellarranged set of base mode tensors like the even and odd vectors used above.

\section{References}

[CBM] Y. Choquet-Bruhat, and V. Moncrief, Future global in time Einsteinian spacetimes with U(1) isometry group Ann. Henri Poincaré 2 (2001) 1007-1064; Non linear stability of einsteinian spacetimes with $U(1)$ isometry group, preprint (gr-qc/0302021).

$[\mathrm{CH}]$ L. Cesari, Asymptotic behavior and stability problems in ordinary differential equations, Springer, 3rd ed. (1971); P. Hartman, Ordinary differential equations, John Wiley (1964).

[EM] G.F.R. Ellis, and M.A.H. MacCallum, A class of homogeneous cosmological models, Commun. Math. Phys. 12 (1969) 108-141.

$[\mathrm{FM}]$ A.E. Fischer, and V. Moncrief, The reduced Einstein equations and the conformal volume collapse of 3-manifolds, Class. Quantum Grav. 18 (2001) 4493-4515; and reference therein.

[HS] J. Hempel, 3-manifolds, Ann. of Math. Studies 86, Princeton University Press (1976); P. Scott, The geometry of 3-manifolds, Bull. London Math. Soc. 15 (1983) 401-487.

[KTH] T. Koike, M. Tanimoto, and A. Hosoya, Compact Homogeneous Universes, J. Math. Phys. 35 (1994) 4855-4888.

[TAU] A. H. Taub, Empty space-times admitting a three-parameter group of motions, Ann. Math. 53 (1952) 472-490.

[TKH] M. Tanimoto, T. Koike, and A. Hosoya, Dynamics of compact homogeneous universes, J. Math. Phys. 38 (1997) 350-368; Hamiltonian structures for compact homogeneous universes, J. Math. Phys. 38 (1997) 6560-6577. 
[TMY] M. Tanimoto, V. Moncrief, and K. Yasuno, Perturbations of spatially closed Bianchi III spacetimes, Class. Quantum Grav. 20 (2003) 1879-1927.

[WE] J. Wainwright, and G.F.R. Ellis, Dynamical Systems in Cosmology, Cambridge University Press (1997).

[YT] K. Yasuno, and M. Tanimoto, Asymptotic behavior of linearly perturbed spatially closed Bianchi III vacuum solutions, in preparation.

[YO] J.W. York Jr., Conformally invariant orthogonal decomposition of symmetric tensors on Riemannian manifolds and the initial-value problem of general relativity, J. Math. Phys. 14 (1973) 456-464.

\section{Errata to the published version}

This article will be published in Contemporary Mathematics of American Mathematical Society. The following is corrections to that published version. (Unfortunately, authors to this publication are not given a chance to make corrections after the final submission.) All the corrections have already been incorporated in the present electronic version, so there is no need to make any change for this article.

1. The line below Eq.(3.14) should read

"where $l=0,1, \cdots, \infty, \quad|m|=1,2, \cdots, \infty, \quad n_{0}=0,1, \cdots,|m|-1 . "$

2. Eq.(2.2) and Eq.(3.1) should be swapped.

Department of Physics, Yale University, New Haven, CT 06511

Current address: Max-Planck-Institut für Gravitationsphysik, Golm 14476, Germany 